\title{
Consideraciones sobre 3.000 partos
}

\section{CAMILO CAPASSO}

El que ha estudiado o estudia los diversos tratados de obstetricia, ve on ellos como los distintos autores ense. ñan basados en estadisticas de sus respectivos paises, la frecuencia de las diferentes presentaciones, el peso y la talla de los fetos a termino, la tre cuencia de intervenciones como apl. cacion de lórceps, cesareas versio nes, y el porcentaje de las diversus distocias etc.

- Personalmente me he siempre pre guntado: se presentarán los mismos porcentajes de presentaciones, disto clas, intervenciones obstéricas. etc. ei nuestras pacientes?

- En esto se basa este modesto traba 16. Son consideracione: sobre 3.000 ratos atendidos yer miembros de $k$ Soctedod Colombian de Obstertcir an el Institulo Colombiane de Segu ios Sociales.

Hubiera querido presentar una $c 0$ suistica maryor ples sólo en la Clinica Primero de Mayo se han atendid. cerca de 10.000 partos; desatortunadr. mente muchas circunstancias me lim. taron a concretarme a 3.000 casos.

1 - Consideraciones generales - Lu nayoria de las pacientes pertenecent - la clase obrera y por ende viver en condrciones anthigienicas, en habua ciones-estrachas, on zutricion deticiente. Un gran porcentcie de ellas tro. baja en condiciones penosas, lo que sumado a su standard. de vida, ha. ce más siqnificativa la estadística presentada.

Alortunadamente la legislación dictada en defensa de la maternidad pro. vee al descanso en el último mes do embarazo, y el ICSS cumpliendo la finalidad legal remunera a la embarazada que descansa durante e! iltimo mes de su gestación.

La atencion obstetrica que brinda el ICSS a sus afiliadas y beneficiarias, similar a la que podría aspirar cual. quier persona, por - medios económi. cos que distrute, balancea en gran parte las deficientes condiciones de vida de la clase obrera. De ahi que me congratule con la Sociedad Colom. bian's de Obstetricic y con las directivas del Sequro, quienes en conjun to han organizado todo lo referente a la atención prendital del pario y puer perio.

Toda paciente escoje su obstetra de contianza y llega al parto después de - haber sido examinada periódica y minuciosamente durante el embarazo.

Ningún examen se les escatima; abrcugralias, reacciones setológicas repetidas, R. H. exarnenes de orina. radiografics, rodiopelvimetrias, en fin cuanto el obstetta considere indispen sable. 
ac se hacen iuntas entre varios es pacilislas para discutur o aconsejar. : conducta obstétrica más convenien. te quizás por todo lo anterior este tra baic arraja datos de mortinatalidad ci.. no tienén nada qué envidiar " $c$ : astadisticas de los mejores hospi. tel mundo.

und totalidal d. 3000 caso:

barazos extraute.

rinos .... . . . . G $\quad$ G $0.20 \%$ Abortos. $=214 \quad .7 .10 \%$ Partos prematuros... $134 \quad 4.47 \%$ Primiparas a término $800-26.66 \%$ Multicaras a término $1.846 \quad 61.54 \%$

Sumas . . $3.000100 .00 \%$

Nota: Los partos de primiparas fue

Unicos.

794

Dobles.

$6.0 .75 \%$

Los partos de mulúparas, fueron:

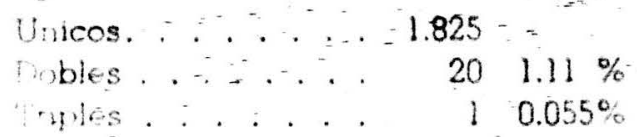

Er relacion con la trecuencia del abatazo extrauterino en los distin10. servicios de obstetricia, he podido tecoger los siguientes dátos:

\$. Kawashita. . . . . . . . . $016 \%$ Manuel L. Pérez. ..... . . . . $0.45 \%$

4. Miller . . . . . . . ... . . $0.66 \%$

A. de Queiroz ... . . . . . $1.00 \%$ Thuestra estadística da sola mente e! ........... $0.20 \%$

Con la irecuencia del aborta, los :rulentes porcentaies: partos

Taussing - 1 aborto para cada 2.5 par. tos en ciudades y 1 aborto para cada 5 partos en el campo.

Nuestra estadistica nos sorprende con el exigu numero de abortos yo que arrola: I abrto parg cada 13 partos. Estoy convencido de que este dato es consecuencin de la teligron que protesan médico y paciente: la católica.

En relación con los partos prematuros:

K. Schultze. . . . . . . . . $6.6 \%$ Murphy y Bowmann . . . . ... $8 . \%$

Nuestra estadística da un $4.47 \%$. Hay que anotar que los porcentajes de Jos anteriores cutores son sacados de hospitales, y el nuestro de pacientes a quienes se somete al más riguraso control-y tratamiento (T B. C.. Sífilis, Toxemias, etc.), desde el embarazo.

Con relación a las presentaciones en OIIA, OIDP, OIIP $y$ on CARA son sensiblemente iguáles. Es de hacer no. tar el aumento considerable de la va. riedad más rara entro las de vértice flecadas o sea la OIDA que arroja un porcentaje tres veces superior al quo tra el doctor Manue! L. Pèrez $(2 \%)$ y 16-veces superior al del doctor $C$. Wassermann $(0.43 \%)$.

Con la presentación de pelvis se comprueba una vez más la importancia de la edad del embarazo como factor etlológico de la presentación.

7 meses de embarazo. $18^{-\%}$

$71 / 2$ meses de embarazo . , 11.1\%

8 meses de embarazo ..... $5.5 \%$

9 meses primiparas : . . . $3 \%$

9 meses multiparas. . ... $2.64 \%$ 
mediada entre -primparas con un $0.25 \%$ y multíparas por un $0.65 \%$, nos da -un $0.38 \%$, porcentaje muy iqual $a$ los de otros países como Inglaterri $0.4 \%$, U.S.A., $0.5 \%$ (De Lee, $0.5 \%, \mathrm{Ma}$. nuel L. Pérez, 0.75).

Peso promedio de los fetos masculinos $y$ temeninos (prematuros y a término) en multíparas $y$ primiparas

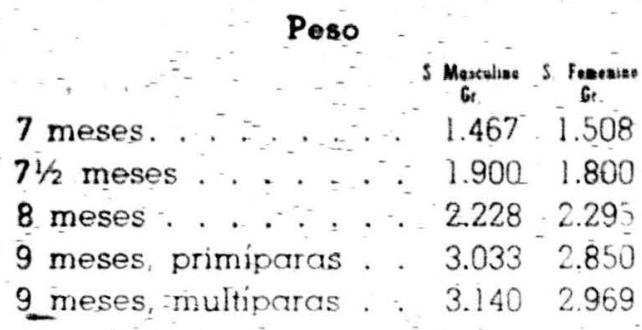

\section{Longitud}

$\begin{array}{lllll}7 \text { meses . . . . . . . } & 40.80 & 40.60 \\ 71 / 2 \text { meses . . . . . } & 43.10 & 43.10 \\ 8 \text { meses . . . } & \ddots & 46.00 & 46.00 \\ 9 \text { meses, primiparas . . } & 49.72 & 48.8 \\ 9 \text { meses, multiparas . . } & 49.50 & 49.00\end{array}$

Porcentaje de fetos masculinos $y$ lemeninos en multiparas y primipara.3

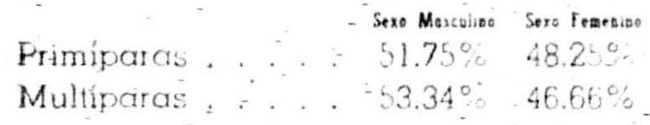

Con relacion al pese y talla lós au. tores tierren los siguientes promedios:

\section{Vinckl y Frankenhauser:}

3.525 Gims. para varones.

3.200 Girms. para mujeres.

Sillman Lanroth:

$$
\begin{aligned}
& -3.391 \text { Gims. para varones. } \\
& 3.327 \text { Grms. para mujeres. }
\end{aligned}
$$

\section{$3.44 \mathrm{i}$ Grms. para varones. \\ 3.323 Grms. para mujeres.}

En nuestra estadística se comprendieron entré los fetos a término aquéllos nacidos de $81 / 2$ meses en adelar. te: ademas hubo discriminacion entre peso de priminacas y multhans

\section{Las primiparas dieron:}

3.033 Gms para los vatunes.
2.850 Grms. para las mujetes.

\section{Las multiparas dieron:}

3.1 40 Grms. para los varones. 2.969 Grms. para las muieres.

Son, pues, letos relativamene más pequeños que los que muestran los autores alemanes, ingleses $y$ argent. nos.

Lo mismo se puede decir con rela- ción a la talla. La mayoria de los autores dan una longitud de 50 Cims. sin distinguir entre telos de primiparas y de multiparas: ni de varones y mujo. res. En nuestra estadistica

\section{Las primiparas dieron:}

\subsection{Cims, prora los varonre. 48.80 Cms para las muieres:}

\section{Las multíparas dieron:}

49.50 Cims. para los varones. 49 Ctms: para las muieres.

Hay que tener en cuenta que' escs pesos y tallas son proporcionclas al tamaño y peso de nuestras mujeres. mas reduendas que las alemmas inglesas, etc. 
y primiparas

\section{Partos}

\begin{tabular}{|c|c|c|}
\hline & Cas. & Percenaleye \\
\hline meses $\ldots$ & 33 & 84.70 \\
\hline $1 / 2$ meses. . & 15 & 83.30 \\
\hline meses. . & 57 & 77.att \\
\hline
\end{tabular}

mins -

$1.462-81.7$

8017

Primiparas तो tel

mino . . . $274 \quad 34.25 \quad 809$

\section{Mortinatalidad}

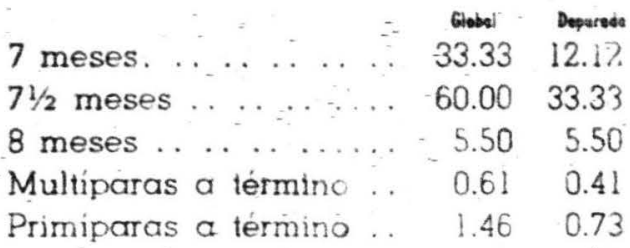

Partos con alguna intervención. prematuros y a término en multíparas y primíparas Mortinatalidad global y depurada en
porcentaje

\section{Partos}

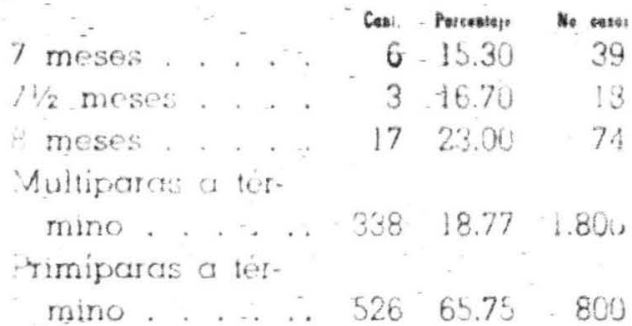

Mortinatalidad

7 méses. . . . . ... 50.00

Deparado

$71 / 2$ meses ... . . . . . . . $\quad .33 .33$

33.33

Multiparas a termino

0.00

33.33

Pimiprias a término

1.45

0.00

1.71
7 meses

Y primiparas

$71 / 2$ meses

Cent. Porcenatey.

Ne. cosen

2. $5.10 \quad 39$

8 meses

5.50

18

Multiparas a tés

mino.

$\begin{array}{lll}163 & 9.06 \quad 1.800\end{array}$

Pmmpras a ter

mino.

\section{Mortinatalidad}

Glabel Boparece

7 meses . . . . . ...... $0.00 \quad 0.00$

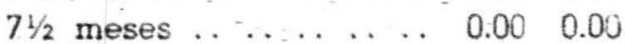

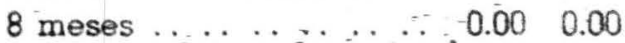

$\begin{array}{lllll}\text { Multíparas a término. } & 0.00 & 0.00\end{array}$

$\begin{array}{lllll}\text { Primiparas a término. } & \ldots & 0.36 & 0.36\end{array}$

Respecto de la episiotomía ningún autor trae estadísticas, ya que es una intervención que se utiliza sistemáticamente para evitar desgarros, muchas veces de tercer grado, una demora innecesaria y a veces perjudicial para ef feto en el período de expulsion.

Nuestra estadística da:

Sobre 800 casos de primiparas:

275 episiotomias sin ninguna etra intervención y 166 episiotomias segut: das de aplicacion de fórceps.

Lo que da un total do 441 episioto mías. Más del $50 \%$ de los casos de donde salta a la vista la importancia de está pequeña mervencion en hues tro medio.

Partos con episiotomía y tórceps (prematuros y a término eñ multipcras y primíparas)

$\begin{array}{rrrrr}7 \text { meses . . . . } & 0.00 & 0.00 & 39 \\ 71 / 2 \text { meses. . . . } & 0 & 0.00 & 18 \\ 8 \text { meses . . . . . } & - & 7 & 9.30 & 71\end{array}$


Prmuaras a tèr-

me...... $166 \quad 20.75 \quad 807$

\section{Mortinatalidad}

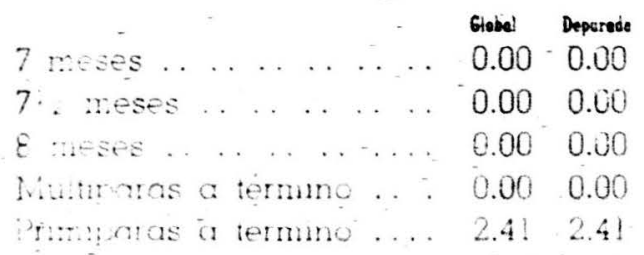

Partos con fórceps únicamente (prematuros y a término en multiparas y primiparas) :

\begin{tabular}{|c|c|c|}
\hline 7 meses... & $\begin{array}{l}\text { Cant. Porreansale } \\
0.00-0.00\end{array}$ & \\
\hline $7 \mathrm{t}_{2}$ neses $\ldots \ldots$ & 0.0 .00 & \\
\hline 8 meses $\therefore . .$. & 1.50 & \\
\hline $\begin{array}{l}\text { Mulmaras a ter- } \\
\text { muro }\end{array}$ & $85 \quad 4.72$ & \\
\hline $\begin{array}{l}\text { Prmul aras or ter } \\
\text { muno. }\end{array}$ & 7,12 & \\
\hline
\end{tabular}

\section{Mortinatalidad}

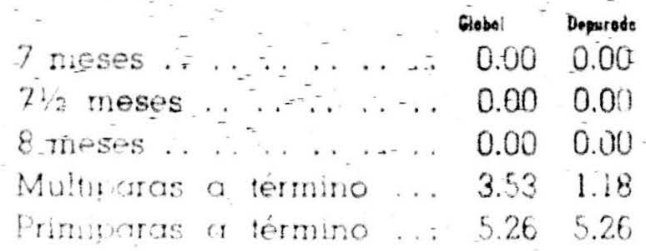

En relcion con el torceps, ningune d. Las estadisticas controntadas hace distinción entre la trecuencia de fór. ces en primiparas y multiparas:

Manuel L. Pérez da . . . . . $4 \%$ H. Helm . . . . . . . . . . 2.65\%

1. de Rezende . . . . . . . . 4.56\%

1. Erbsot . . . . . . . . . 5.38\%

I. A. Gabastou . . . . . . . $2.8 \%$

N. A. Yanzon ........ $4.4 \%$
Nuestra estadística da:

Primíparas, sobre 800 partos:

Fórceps y episiotomía . $-166 \quad 20.75 \%$ Forceps sin episiotomia $\quad-57 \quad 7.125 \%$

Lo que stima 223 aphcocones $d$ forcepen los 800 casos a san un por centere de $27.87 \%$

Muinaras sobre 1.800 partos:

Forceps y epistotomia. . . 37.2 .05 Fórçeps $\sin$ episiotomia. . $85^{\circ} 4.72$

Lo que suma 122 forceps en 1.800 partos de multiparas, lo que eleva el porcentaje a $6.77 \%$.

Sumando los casos de multiparas y primiparas dan 2.600 parlos y los for ceps aplicados en primiparas y multiparas suman 345,10 que da un porcentaje de $13.27 \%$.

Este aumento se debe casi exclus: vamente a la enorme cantidad de for ceps profilácticos que se aplicaron:

En multiparas . . . . . . En primiparas . . . . . . . . . . 105

to cual suma 157 fórceps y como el total de ellos tue de 345, dio un por centaje de $45.5 \%$ de lorcess profilac: ticos.

Relacionando el fórceps con la mor tinatalidad, tampoco en esto las esta disticas hacen - diferenciacion entre mortinatalidad con-fórceps en primparas $y$ multiparas

Sach da $. \ldots . . . . . .2 .81 \%$

H. Helm, da . . . . . . $53 \%$

J. Erbslch, da . . . . . . . . $\quad-5.81 \%$

M. A. Novey, da . . . . . $5.96 \%$

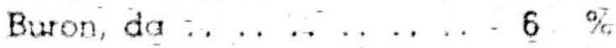


A Peralta Ramos relacionándolo con el plano de aplicación, da:

Por encima del primer plano

de Hodge

Entre el primet y sogurido ple

and hotio

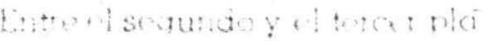

d. itsit

$137^{\circ}$

10.1

9.8

Nuestra madiater ensetia

\section{Primiparas}

Forceps con episioloma en $160 \mathrm{ca}$ sos:

4 mortinatos o- sea un $2.41 \%$.

Forceps sin episiotomia en 57 cassos.

3 mortinatos o sea $5.26^{\circ}$.

\section{Multiparas}

Forceps con episiotomias, en $37 \mathrm{ca}$ sos.

Ningún mortinato.

Forceps sin episiotomía, en 85 caso.

1 mortinato y 2 fetos muertos y-macercados:

Mortinatalidari global, $3.53 \%$. Mor. finatalidad depurada, $2.3 \%$.

Sumando las aplicaciones de to ceps en multiparas y primparas se obtiene un total de 345 forceps. cou 8 mortinatos y 2 fetos muertos y macera dòs.

La mortinatalidad global fue, pues, de $2.9 \%$.

Y la mortinatalidad depurada, de $2.3 \%$.

Tenemos entonces cast $2 \frac{1}{2}$ veces más cantidad de aplicación de fórceps con una motinatalidad que varía de dos a cuatro veces menos con los do.

\section{c}

areas ces

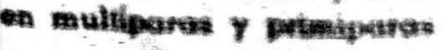

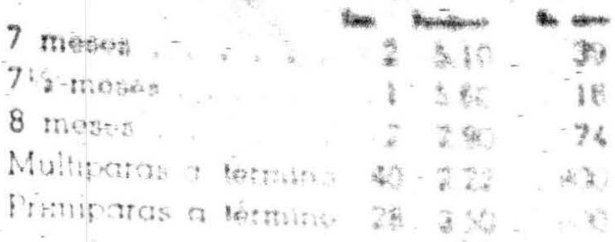

\section{Mortinatalldad}

$\begin{array}{lllllll}7 \text { meses } \ldots & \ldots & \ldots & \ldots & 50.00 & 50.00 \\ 71 / 2 \text { meses } & \ldots & \ldots & \ldots & \ldots & 0.00 & 0.00 \\ 8 \text { meses } \ldots & \ldots & \ldots & \ldots & \ldots & 0.00 & 0.00 \\ \text { Multiparas a termino } & . & 2.50 & 2.50 \\ \text { Primíparas a término } & \ldots & 3.93 & 3.93\end{array}$

Todas las cesareas fueron segmenia. rias y transperitoneales.

Aqui tampoco los autores hacen di. terenclacion entre frecuencia de cesci. reas en primiparas y multiparas.

J. Frigyesi .

P. Felweg . . . . . . . . . . . . $5.8 \%$

T. Monygomery . . . . . $1.6 \%$

Schumamn ......... 25\%

Nuestra estadistica da:

Primiparas sobre 800 partos $28 \mathrm{ce}$. sarecs, un $3.5 \%$.

Multiparas, sobre 1.800 casos, 40 cesareas, un $2.22 \%$

Un tolal de 68 cesáreás subre 2.600 partos, lo que da un $2.65 \%$.

Este porcentaje es sensiblemente igual a los anteriormente chados.

Con relación a la mortinatalidad:

1. P. Greenhill .. . . . . . , $0.8 \%$ Selitzy . . . . . . . . . . $2.7 \%$ Manuel L. Péréz . . .......... $3 \%$ 
Primparas, 1 mortmato.

Multiparas, 1 mortinato.

68 cesáreas con 2 mortinatos dan un porcentaje de $3.4^{\circ}$, también sensible mente iqual a los anteriores citados.

Partos con versión interna prematuros y a término en primiparas y multiparas

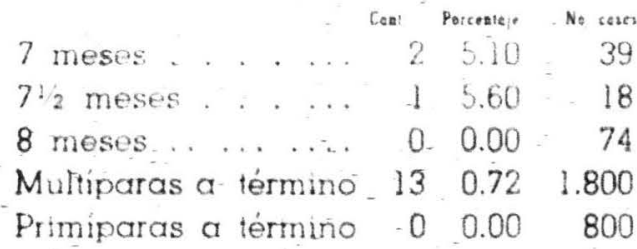

7 meses

100.00

$71 / 2$ meses

100.00

8 méses.

0.00

100.00

Multiparas a termine

7.69

Primiparas a jermino.

Promedio de duración del trabajo en horas. prematuros y a término en primiparas y multiparas.

N. B. En el próximo número se publicarán los cuadros correspondientes a los fórcéps aplicados y a las cesáreas practicadas.

\section{OCTOSPASMOL \\ (ANTIRSPASMODICO)}

COMPOSICION:

Clorhidrato de Papaverina,

Clorhidrato de Octina, bifenil-aceticodietilamino-etanoletérico. Dimetilamino-antipirina-dialilharbiturato.

Ampollas de 1 cc.

Gotas, frasco de $12 \mathrm{cc}$.

Espasmos gastro-intestinales; espasmos vias urinarias: espasmos coronarios, etc.

\section{Laboratorios "Hormona" Colombia, S. A.} Apartado aéreo 4092 . Bogotá. 